\title{
“Workshop” sobre disfunção das glândulas de Meibomius
}

\author{
Workshop on meibomian gland dysfunction
}

José Álvaro Pereira Gomes ${ }^{1}$, Eduardo M. Rocha ${ }^{2}$, Denise de Freitas ${ }^{3}$, David A. Sullivan ${ }^{4}$

A disfunção das glândulas meibomianas (DGM) é uma das doenças mais comuns da prática oftalmológica e está associada a várias alterações da superfície ocular e ao olho seco. Apesar da sua importância no dia-a-dia do oftalmologista, DGM não havia recebido a atenção merecida na literatura médica nacional e internacional. Nesse contexto, a "Tear Filme \& Ocular Surface Society" (TFOS) recrutou mais de 50 especialistas no assunto e organizou um "workshop" Internacional sobre DGM(1). Esse "workshop", que teve mais de dois anos de duração, apresentou como objetivo principal estabelecer um consenso em relação à definição, classificação, fisiopatologia, epidemiologia, diagnóstico e terapia para esta doença, permitindo uma padronização de condutas necessária e bem-vinda para todos os oftalmologistas. Pela sua importância e valor, o "workshop" foi publicado num suplemento na prestigiosa revista Investigative Ophthalmology and Visual Science (IOVS) nesse ano de $2011^{(2)}$ e teve o seu resumo traduzido para o português e publicado em anexo a este editorial do ABO.

A DGM é uma anormalidade crônica e difusa das glândulas meibomianas, caracterizada por obstrução do ducto terminal e/ou mudança quantitativa/qualitativa da secreção glandular, que consequentemente resulta em alteração do filme lacrimal. Essa alteração traduz-se em inflamação clínica aparente da superfície ocular e sintomas como irritação ocular e embaçamento visual, que podem limitar as atividades dos pacientes ${ }^{(3)}$.

A classificação da DGM baseia-se na secreção das glândulas meibomianas e pode ser dividida em dois grupos: baixa secreção e alta secreção. O estado de baixa secreção pode ser subclassificado em hiposecretor e em obstrutivo, podendo ser este último cicatricial e não-cicatricial(4).

Primariamente, a DGM de baixa secreção é causada por obstrução do ducto terminal devido à hiperceratinização do epitélio ductal e à aumentada viscosidade meibomiana, processo influenciado por fatores como idade, sexo, distúrbios hormonais, medicação tópica, dentre outros. A obstrução meibomiana pode originar dilatação cística intraglandular, atrofia e diminuição da secreção lipídica. Como resultado, há aumento da evaporação da lágrima, hiperosmolaridade, instabilidade lacrimal, crescimento bacteriano na margem palpebral e inflamação da superfície ocular(5).

A prevalência da DGM parece ser maior na população asiática (mais de 60\%), enquanto varia de 3,5 a 19,9\% entre os caucasianos. Inúmeros fatores podem ser associados à DGM, coexistindo ou participando da patogênese desta doença, tais como blefarite anterior, uso de lente de contato(6,7), infestação por Demodex folliculorum ${ }^{(8-11)}$, olho seco ${ }^{(12,13)}$, deficiência de andrógeno, menopausa, idade avançada, síndrome de Sjögren, dislipidemia, psoríase ${ }^{(14)}$, atopia ${ }^{(15)}$, rosácea ${ }^{(16,17)}$, hipertensão, hiperplasia prostática benigna ${ }^{(18)}(\mathrm{HPB})$, uso de antiandrógeno, terapia hormonal pós-menopausa, uso de anti-histamínicos, antidepressivos e retinóide. O uso de ômega-3 parece ser um fator de proteção(19).

Para o diagnóstico de DGM, há a recomendação de que sejam feitos os seguintes exames: 1) administração de um questionário de sintomas; 2) medida da frequência e do intervalo do piscar; 3) medida da altura do menisco lacrimal inferior; 4) medida da osmolaridade do filme lacrimal; 5) medida do tempo de ruptura do filme lacrimal (BUT) e do Índice de Proteção Ocular; 6) classificação da coloração conjuntival e corneana com corante vital; 7) teste de Schirmer ou teste vermelho de fenol. Caso haja anormalidade nos testes 1, 4, 5 e 6, há evidência parcial da presença de olho seco genérico. Evidência de olho seco por deficiência aquosa pode ser obtida pela medida do fluxo de filme lacrimal ou pela altura do menisco lacrimal ou pelo teste de Schirmer. Caso não se tenha caracterizado DGM, a sequência diagnóstica pode ser continuada da seguinte forma: 8) a- quantificação das características morfológicas da pálpebra, b- quantificação da expressão meibomiana e avaliação da qualidade da secreção e c- quantificação da saída de secreção(20) (meibografia). Se os testes sugerirem olho seco genérico e os testes de fluxo e de volume lacrimal forem normais, olho seco evaporativo torna-se evidente e a quantificação da DGM indicará a contribuição das glândulas meibomianas na alteração do filme lacrimal e da superfície ocular(21).

O tratamento da DGM foi padronizado para cada estágio: 1- higiene palpebral, compressas mornas e expressão das glândulas com massagem; 2- os anteriores, adicionados de uso de ômega-32(22), lubrificante artificial, azitromicina

${ }^{1}$ Physician, Department of Ophthalmology of Federal University of São Paulo - UNIFESP - São Paulo

(SP), Brazil.
2 Physician, Department of Ophthalmology, Otorhinolaryngology, and Head and Neck Surgery -

Universidade de São Paulo, Ribeirão Preto (SP), Brazil.

${ }_{3}$ Associate Professor, Chief of Department of Ophthalmology of Federal University of São Paulo -

UNIFESP - São Paulo (SP), Brazil.

${ }^{4}$ Senior Scientist of Schepens Eye Research Institute, Associate Professor, Department of Ophthal-

mology, Harvard Medical School, President, Tear Film \& Ocular Surface Society.
}

Funding: No specific financial support was available for this study.

Disclosure of potential conflicts of interest: Gomes JAP, Consultant (Allergan), Recipient (Alcon, Natura); Rocha EM, None; de Freitas D, None; Sullivan DA, Consultant (Pfizer), Financial Support (Alcon), Board Member (Singularis, TearLab), Personal Financial Interest (Singularis, TearLab). 


\section{Disfunção da glândula meibomiana O que é, por que ocorre e como pode ser tratada?}

Kelly K. Nichols, Gary N. Foulks, Anthony J. Bron e David A. Sullivan, em nome dos participantes do "workshop" internacional sobre disfunção da glândula meibomiana

\section{Introdução}

Embora a disfunção da glândula meibomiana (DGM) seja uma doença comum, crônica, debilitante, que influencia a saúde e 0 bem-estar de milhões de pessoas no mundo, não existe um consenso global quanto à sua definição, classificação, diagnóstico ou terapia. Para consegui-lo, a Tear Film \& Ocular Surface Society (TFOS) patrocinou o "workshop" internacional sobre disfunção da glândula meibomiana (www.TearFilm.org).

Foram necessários dois anos para concluir este "workshop", que contou com a participação de mais de 50 peritos internacionais.

0 relatório do "workshop" é agora publicado no jornal IOVS vol. 52. Salienta-se adiante alguns pontos com as respectivas recomendações.

\section{0 que é DGM?}

O "workshop" definiu a DGM da forma seguinte:

A disfunção da glândula meibomiana (DGM) é uma anomalia crônica, difusa das glândulas meibomianas, geralmente caracterizada por uma obstrução do ducto terminal e/ou alterações qualitativas/quantitativas da secreção glandular. Isso pode provocar uma alteração do filme lacrimal, sintomas de irritação ocular, inflamação clinicamente aparente e uma doença da superficie ocular. A DGM é classificada em duas categorias principais baseadas na secreção das glândulas meibomianas:

1) Estados de baixa secreção - a causa mais frequente, $e$

2) Estados de alta secreção (Figura 1).

Finalmente, a DGM pode causar alterações do filme lacrimal, sintomas de irritação ocular, inflamação da superficie ocular e olho seco.

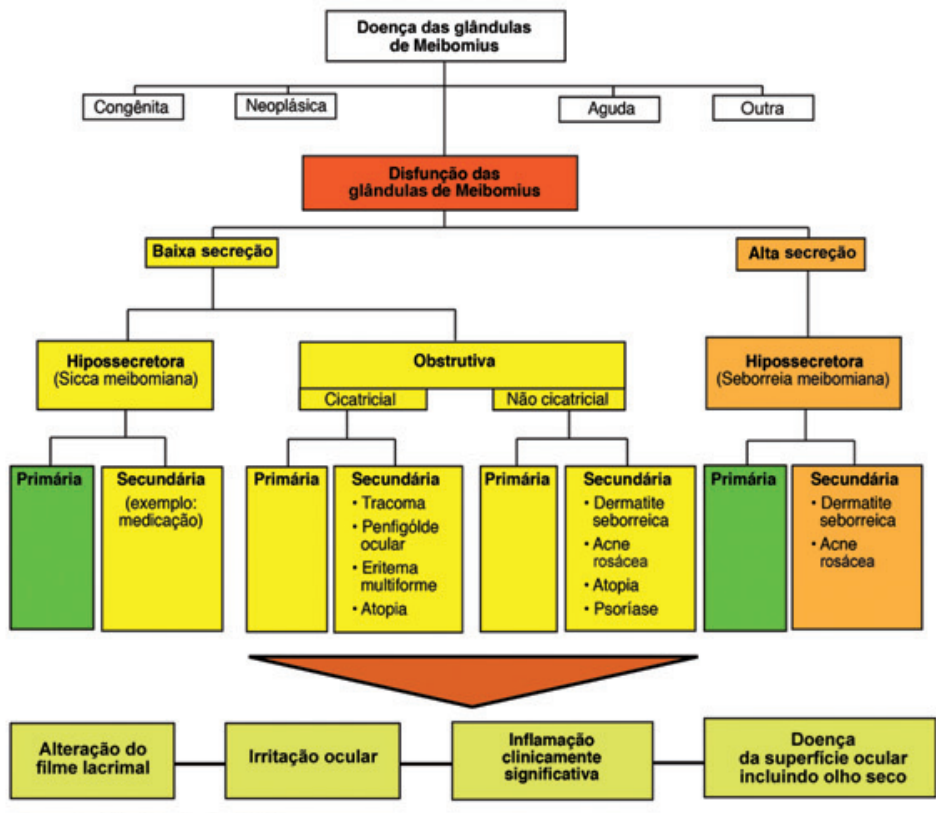

Figura 1. Classificação da DGM

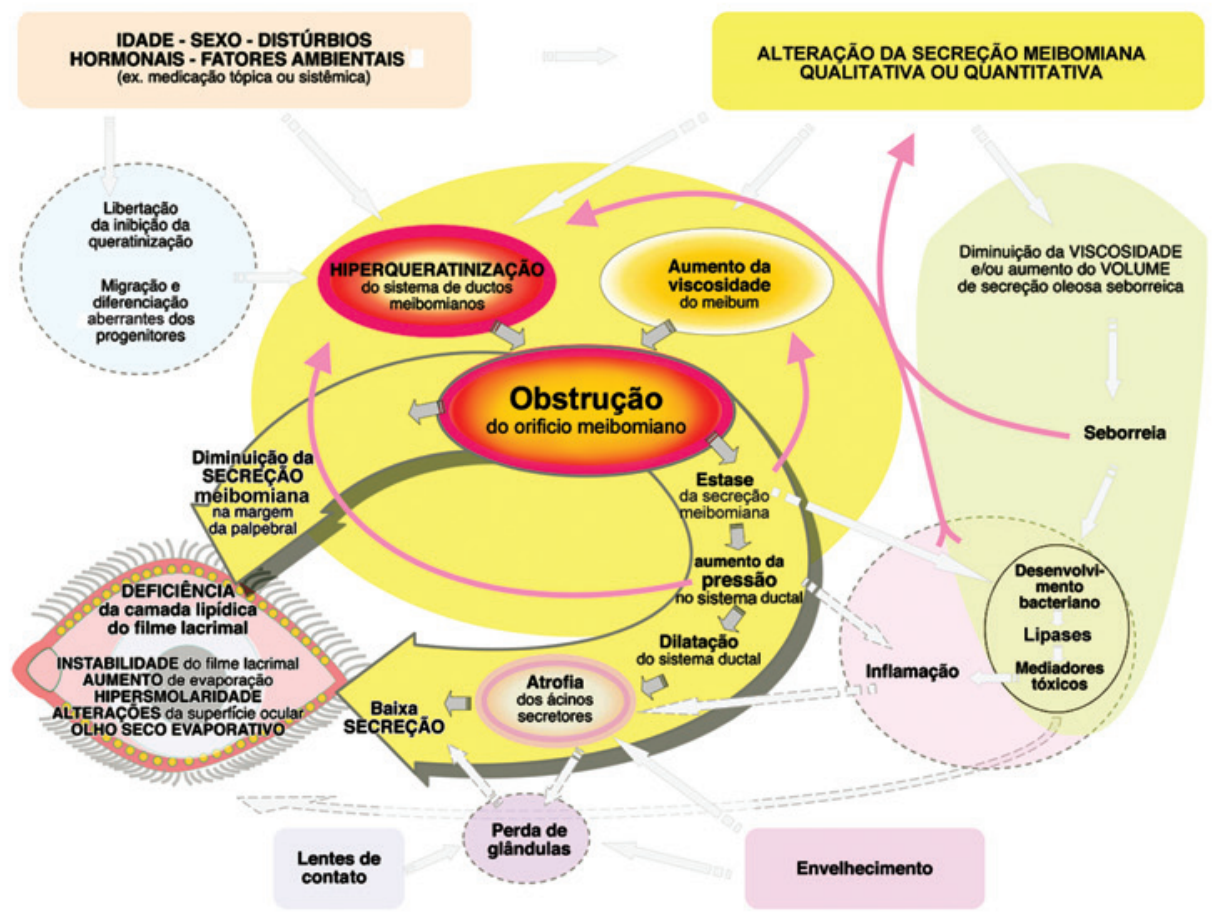

Figura 2. Patofisiologia da DGM obstrutiva 
Por que ocorre a DGM?

A DGM obstrutiva, de baixa secreção, é causada principalmente pela obstrução do ducto terminal, devido à hiperqueratinização do epitélio ductal, a restos de células queratinizadas e ao aumento da viscosidade da secreção meibomiana (Figura 2). Uma forma cicatricial é também importante.

O processo de obstrução é influenciado por fatores endógenos como a idade, o sexo e distúrbios hormonais, bem como por agentes exógenos como agentes sistêmicos (por exemplo retinóides) e eventualmente o uso de lentes de contato. Associações secundárias importantes são as doenças da pele (por exemplo: acne rosácea; dermatite atópica e seborreica) e conjuntivite cicatricial (por exemplo, eritema multiforme; tracoma). A obstrução pode causar dilatação cística intraglandular, atrofia das células meibomianas, perda da glândula e baixa secreção, efeitos que não envolvem normalmente inflamação.

O resultado final é uma disponibilidade reduzida de secreção meibomiana na margem da pálpebra e na camada lipídica do filme lacrimal, podendo causar a instabilidade do filme lacrimal, aumento da evaporação, hiperosmolaridade lacrimal, olho seco evaporativo bem como inflamação e danos da superficie ocular. De um modo geral, a DGM é uma patologia importante, subestimada e é muito provavelmente a causa mais frequente da doença do olho seco.

\section{Como pode ser tratada a DGM?}

Apresenta-se adiante uma abordagem com base nas evidências para a gestão da DGM. Em cada nível de tratamento, a falta de resposta à terapia faz avançar o tratamento para o nível seguinte. Um sinal [ \pm ] significa que as provas para apoiar o uso do tratamento são limitadas ou emergentes, por conseguinte o uso deverá ser baseado no julgamento clínico. Um sinal $[+]$ indica que o tratamento é apoiado por evidência nessa fase da doença. A qualidade da secreção meibomiana expressa e a expressibilidade da secreção meibomiana são fatores chave na avaliação clínica da DGM. Mais detalhes sobre a gestão da DGM e doenças relacionadas com a DGM são apresentados no relatório completo.

\begin{tabular}{|c|c|c|}
\hline FASE & DESCRIÇÃO CLINICA & TRATAMENTO \\
\hline FASE 1 & $\begin{array}{l}\text { Assintomática: } \\
\text { Sem sintomas de desconforto ocular, prurido ou fotofobia } \\
\text { Sinais clinicos da DGM baseados na expressão da glândula } \\
\text { Secreções minimamente alteradas: Grau }>2-<4 \\
\text { Expressividade: } 1 \\
\text { Sem coloração da superficie ocular }\end{array}$ & $\begin{array}{l}\text { Informar o paciente sobre a DGM, o impacto potencial da } \\
\text { dieta e os efeitos dos ambientes de trabalho/casa na } \\
\text { evaporação lacrimal, e o possivel efeito de secura de certas } \\
\text { medicações sistémicas } \\
\pm \text { Considerar a higiene das pálpebras incluindo aquecimento/ } \\
\text { expressão como descrito abaixo }\end{array}$ \\
\hline FASE 2 & $\begin{array}{l}\text { Sintomas mínimos a leves de desconforto ocular, prurido } \\
\text { ou fotofobia } \\
\text { DGM mínima a leve } \\
\text { Caracteristicas difusas na margem da pálpebra } \\
\text { Secreç̋̋es ligeiramente alteradas: Grau }>4-<8 \\
\text { Expressividade: } 1 \\
\text { Sem a ligeira coloraçăo da superficie ocular } \\
\text { [DEWS 0-7; Oxford 0-3] }\end{array}$ & $\begin{array}{l}\text { Aconselhar o paciente a melhorar a umidade ambiente; } \\
\text { optimizar os postos de trabalho e aumentar a ingestão de } \\
\text { ácidos gordos omega-3 na dieta }( \pm \text { ). } \\
\text { Instituir higiene da pálpebra com aquecimento da pálpebra } \\
\text { (no mínimo quatro minutos, uma ou duas vezes por dia) } \\
\text { seguido de massagem moderada a firme e expressão das } \\
\text { secreçőes das GM (+). } \\
\text { Tudo acima referido, mais: } \\
\pm \text { Lubrificantes artificiais (para uso frequente, preferir sem- } \\
\text { conservantes) } \\
\pm \text { Lubrificante emoliente tópico ou "spray" lipossomal } \\
\pm \text { Azitromicina tópica } \\
\pm \text { Considerar derivados orais de tetraciclina }\end{array}$ \\
\hline FASE 3 & $\begin{array}{l}\text { Sintomas moderados de desconforto ocular, prurido ou } \\
\text { fotofobia com limitação da atividade } \\
\text { DGM moderada } \\
\uparrow \text { caracteristicas da margem da pálpebra (obstrução, } \\
\text { vascularidade) } \\
\text { Secreções moderadamente alteradas: Grau }>8-<13 \\
\text { Expressividade: } 2 \\
\text { Coloração leve a moderada da córnea periférica e conjuntiva, } \\
\text { frequentemente inferior [DEWS } 8-23 ; \text { Oxford } 4-10]\end{array}$ & $\begin{array}{l}\text { Tudo acima referido, mais: } \\
\text { + Derivados orais da tetraciclina } \\
\pm \text { Pomada lubrificante ao deitar } \\
\pm \text { Terapia anti-inflamatória para olho seco, como indicado }\end{array}$ \\
\hline \multirow[t]{2}{*}{ FASE 4} & $\begin{array}{l}\text { Sintomas marcados de desconforto ocular, prurido ou } \\
\text { fotofobia com limitação definitiva das atividades }\end{array}$ & $\begin{array}{l}\text { Tudo acima referido, mais: } \\
\text { + Terapia anti-inflamatória para olho seco }\end{array}$ \\
\hline & $\begin{array}{l}\text { DGM grave } \\
\uparrow \text { características da margem da pálpebra (perda, deslocação) } \\
\text { Secreçôes gravemente alteradas: Grau >13 } \\
\text { Expressividade: } 3 \\
\text { Maior coloração da córnea e da conjuntiva, incluindo } \\
\text { coloraçăo central [DEWS } 24-33 ; \text { Oxford } 11-15 \text { ] } \\
\uparrow \text { Sinais de inflamação: ex. uma hiperémia conjuntival } \\
\text { moderada, flictenulose }\end{array}$ & $\begin{array}{l}\text { Chave: } \\
\text { A qualidade da secreção meibomiana é avaliada em cada uma } \\
\text { das } 8 \text { glândulas do terço central da pálpebra inferior numa escala } \\
\text { de } 0-3 \text { para cada glândula: } 0=\text { secreção meibomiana clara; } 1= \\
\text { secreçäo meibomiana nublada; } 2=\text { nublada com detritos } \\
\text { (granulado); } 3=\text { espessa como pasta de dentes [variação de } 0-24 \text { ] } \\
\text { A expressividade da secreção meibomiana é avaliada em } 5 \\
\text { glândulas: } 0=\text { todas as glândulas expressivas; } 1=3-4 \text { glândulas } \\
\text { expressivas; } 2=1-2 \text { glândulas expressivas; } 3=\text { nenhuma glândula } \\
\text { expressiva. Pode ser avaliada na pálpebra inferior ou superior. } \\
\text { Os niveis de coloração numérica referem-se a um nivel } \\
\text { resumido de coloração da córnea e da conjuntiva exposta. O } \\
\text { esquema de Oxford tem uma escala de } 0-15 \text { e o de DEWS } \\
\text { uma escala de } 0-33 \text {. }\end{array}$ \\
\hline
\end{tabular}

Agradecimentos: Agradecemos Michelle Dalton (www.dalton-and-associates.com) e Sabrina Zappia e CITYNet (uww.citynetonline.it) pela sua assistência profissional neste resumo do relatório do "workshop". Uma lista dos participantes do "workshop" pode ser encontrada em: www.tearfilm.org/mgdworkshop/Mgd_Subcommittees.html

Laboratórios Théa (www.laboratoires-thea.com) patrocinaram a tradução desteanexo, ARVO (www.arvo.org) publicou o relatório eTFOS (www.TearFilm.org) patrocinou o encontro sobre DGM. 
tópica ${ }^{(23,24)}$, lubrificante tópico emoliente ou "spray" lipossomal e derivados de tetraciclina orais"(25); 3 - os anteriores + pomada lubrificante ao dormir; 4- os anteriores e terapia anti-inflamatória para olho seco. Para a doença plus, recomendam-se os seguintes tratamentos: 1) inflamação exacerbada da superfície ocular - pulso de esteróide fraco; 2) ceratinização mucosa - lente de contato terapêutica; 3) ceratite flictenular esteróide; 4) triquíase - epilação, crioterapia; 5) calázio - esteróide intralesional ou exérese; 6) blefarite anterior - antibiótico tópico (com ou sem esteróide); 7) blefarite anterior associada ao Demodex - "tea tree oil"(26).

Com este editorial, o ABO e a TFOS, decidiram introduzir esses importantes resultados e compartilhar com todos os oftalmologistas estas informações.

\section{REFERÊNCIAS}

1. MGD Redefined: International Workshop on Meibomian gland dysfunction report [Internet]. Boston: The Tear Film \& Ocular Surface Society (TFOS); March 31, 2011. [cited 2011 May 31]. Available from: www.tearfilm.org/mgdworkshop/index.html

2. Nichols KK. The international workshop on meibomian gland dysfunction: introduction. Invest Ophthalmol Vis Sci. 2011;52(4):1917-21.

3. Nichols KK, Foulks GN, Bron AJ, Glasgow BJ, Dogru M, Tsubota K, et al. The international workshop on meibomian gland dysfunction: executive summary. Invest Ophthalmol Vis Sci. 2011;52(4):1922-9.

4. Nelson JD, Shimazaki J, Benitez-del-Castilo JM, Craig JP, McCulley JP, Den S, et al. The international workshop on meibomian gland dysfunction: report of the definition and classification subcommittee. Invest Ophthalmol Vis Sci. 2011; 52(4):1930-7.

5. Knop E, Knop N, Millar T, Obata H, Sullivan DA. The international workshop on meibomian gland dysfunction: report of the subcommittee on anatomy, physiology, and pathophysiology of the meibomian gland. Invest Ophthalmol Vis Sci. 2011; 52(4):1938-78.

6. McCulley J P, Shine WE. Eyelid disorders: the meibomian gland, blepharitis, and contact lenses. Eye Contact Lens. 2003; 29(1 Suppl): S93-5; discussion S115-8, S192-4.

7. Lemp MA, Bielory L. Contact lenses and associated anterior segment disorders: dry eye disease, blepharitis, and allergy. Immunol Allergy Clin North Am. 2008;28(1):105-17, vi-vii.

8. Liu J, Sheha H, Tseng SC. Pathogenic role of Demodex mites in blepharitis. Curr Opin Allergy Clin Immunol. 2010;10(5):505-10.

9. Kim JT, Lee SH, Chun YS, Kim JC. Tear cytokines and chemokines in patients with Demodex blepharitis. Cytokine. 2011;53(1):94-9.

10. Filho PA, Hazarbassanov RM, Grisolia AB, Pazos HB, Kaiserman I, Gomes JA. The efficacy of oral ivermectin for the treatment of chronic blepharitis in patients tested positive for Demodex spp. Br J Ophthalmol. 2011;95(6):893-5.

11. Divani S, Barpakis K, Kapsalas D. Chronic blepharitis caused by Demodex folliculorum mites. Cytopathology. 2009;20(5):343-4.

12. Bernardes TF, Bonfioli A A. Blepharitis. Semin Ophthalmol. 2010;25(3):79-83.

13. Brewitt H, Kaercher T, Rufer F. [Dry eye and blepharitis]. Klin Monbl Augenheilkd. 2008; 225(2):R15-32, quiz R33. German.

14. Zhu F, Tao JP. Bilateral upper and lower eyelid severe psoriasiform blepharitis: case report and review of literature. Ophthal Plast Reconstr Surg. 2011 April 1.[Epub ahead of print].
15. Asano-Kato N, Fukagawa K, Tsubota K, Urayama K, Takahashi S, Fujishima H. Quantitative evaluation of atopic blepharitis by scoring of eyelid conditions and measuring the water content of the skin and evaporation from the eyelid surface. Cornea. 2001;20(3): 255-9.

16. Neiberg M N, Sowka J. Phlyctenular keratoconjunctivitis in a patient with Staphylococcal blepharitis and ocular rosacea. Optometry. 2008:79(3):133-7.

17. Jackson WB. Blepharitis: current strategies for diagnosis and management. Can J Ophthalmol. 2008;43(2):170-9. Comment in: Can J Ophthalmol. 2008;43(4):485.

18. Nemet AY, Vinker S, Kaiserman I. Associated morbidity of blepharitis. Ophthalmology. 2011;118(6):1062-8

19. Schaumberg DA, Nichols JJ, Papas EB, Tong L, Uchino M, Nichols KK. The international workshop on meibomian gland dysfunction: report of the subcommittee on the epidemiology of, and associated risk factors for, MGD. Invest Ophthalmol Vis Sci. 2011; 52(4):1994-2005.

20. McCann LC, Tomlinson A, Pearce El, Diaper C. Tear and meibomian gland function in blepharitis and normals. Eye Contact Lens. 2009;35(4): 203-8.

21. Tomlinson A, Bron AJ, Korb DR, Amano S, Paugh JR, Pearce El, Yee R, et al. The international workshop on meibomian gland dysfunction: report of the diagnosis subcommittee. Invest Ophthalmol Vis Sci. 2011:52(4):2006-49.

22. Macsai MS. The role of omega-3 dietary supplementation in blepharitis and meibomian gland dysfunction (an AOS thesis). Trans Am Ophthalmol Soc. 2008;106:336-56.

23. Haque R, Torkidsen GL, Brubaker K, Zink RC, Kowalski RP, Mah FS, et al. Multicenter openlabel study evaluating the efficacy of azithromycin ophthalmic solution $1 \%$ on the signs and symptoms of subjects with blepharitis. Cornea. 2010;29(8):871-7.

24. Luchs J. Efficacy of topical azithromycin ophthalmic solution $1 \%$ in the treatment of posterior blepharitis. Adv Ther. 2008;25(9):858-70.

25. Ta CN, Shine WE, McCulley JP, Pandya A, Trattler W, Norbury JW. Effects of minocycline on the ocular flora of patients with acne rosacea or seborrheic blepharitis. Cornea. 2003; 22(6):545-8,

26. Geerling G, Tauber J, Baudouin C, Goto E, Matsumoto Y, O'Brien T, et al. The international workshop on meibomian gland dysfunction: report of the subcommittee on management and treatment of meibomian gland dysfunction. Invest Ophthalmol Vis Sci. 2011; 52(4):2050-64. 